\title{
Les grandes stations de pompage
}

\author{
Large pumping plants \\ par R. Barreau \\ Société DEGREMONT
}

Degrémont is specialised in large treatment works for sewage water and drinking water. Especially for large drinking water plants, Degrémont has to supply raw andlor treated water pumping plants. Numerous criteria have to be taken into consideration while designing a pumping plant, like for instance :

- water temperature, with influence on viscosity, outer condensing on pipes,

- dissolved salts and gas content,

- suspended solids, among them big materials, like larger pieces of wood used during construction in a $4 \mathrm{~m}^{3} / \mathrm{s}$ pumping plant of the YangTse river in ChongQing, China, where we had to clean once again a $200 \mathrm{~m}$ inlet pipe containing pieces of wood as big as $0.6 \times 0.15 \times 0.15 \mathrm{~m}$.

These materials were linked to a poor cleaning of the pipe, but under some circunstances some problems can be linked to the raw water itself. In Cisadane, Indonesia, plastic bags are very commonly found in the raw water due to urban pollution. These plastic bags caused 6 large pumps to be stopped for cleaning after as little as 6 hours duty. The solution was long and difficult to find, the customer has to install mamual screens that he first did not consider ; these screens were initially cleaned by drivers until a scraper was installed.

\section{PRÉAMBULE}

Traiter l'eau c'est mettre en place des procédés où l'aspect hydraulique est prépondérant, car il s'agit de faire transiter l'eau dans plusieurs ouvrages en général en série, pour la rendre potable.

Pour cela il faut d'abord extraire par pompage l'eau de son milieu naturel et l'amener au lieu où les procédés de traitement sont mis en application. Les postes de pompage d'eau brute sont systématiques.

Pour distribuer l'eau potable depuis le réservoir d'eau traitée, il faut soit alimenter un château d'eau, soit mettre en pression un réseau de distribution et là encore un pompage, en général intégré dans l'installation de traitement des eaux, est incontournable.

Dans ces deux types de postes de pompage, les critères hydrauliques permettant une bonne conception des équipements sont essentiels et nous allons voir que certaines incertitudes peuvent avoir des conséquences importantes sur le bon fonctionnement de l'installation.

\section{II — LA QUALITÉ DE L'EAU À POMPER}

Les eaux brutes à pomper sont très différentes d'une contrée à une autre.

Par ailleurs, on peut en distinguer deux types : les eaux de forage issues des nappes phréatiques, et les eaux de surface provenant des fleuves ou rivières.
Un certain nombre de critères les caractérisent qui doivent être pris en considération dans l'étude du poste de pompage. Nous en citerons quelques-uns :

- la température : on constate des différences importantes entre la température des eaux de forage qui peut être très souvent de l'ordre de $10^{\circ} \mathrm{C}$ ou moins et celle des eaux de surface qui peut dépasser les $20^{\circ} \mathrm{C}$ dans les pays chauds.

La température influe sur la viscosité et par conséquent sur les pertes de charge dans les réseaux. Un phénomène important créé par les basses températures d'eau est une condensation constatée sur l'extérieur des conduites à l'air libre lorsque la température du local de pompage est supérieure à celle de l'eau. On constate ces phénomènes après la mise en service, et dans un certain nombre des cas, une déshumidification est alors souvent nécessaire pour éviter ce phénomène.

- les sels et gaz dissous ou non dans l'eau : ils peuvent créer des actions corrosives dans les équipements, en ayant le même effet que la cavitation, c'est-à-dire que des bulles de gaz peuvent attaquer les roues ou la volute des pompes. Le dégazage d'une eau avant pompage est naturellement effectué dans les bâches d'aspiration des pompes lorsque les préconisations des normes sont respectées.

- les matières en suspension peuvent fortement gêner le bon fonctionnement des pompes, et on peut en rencontrer plusieurs types, de plus ou moins grosse granulométrie.

Qui n'a pas constaté au démarrage d'un pompage des bruits anormaux dans une pompe ? 


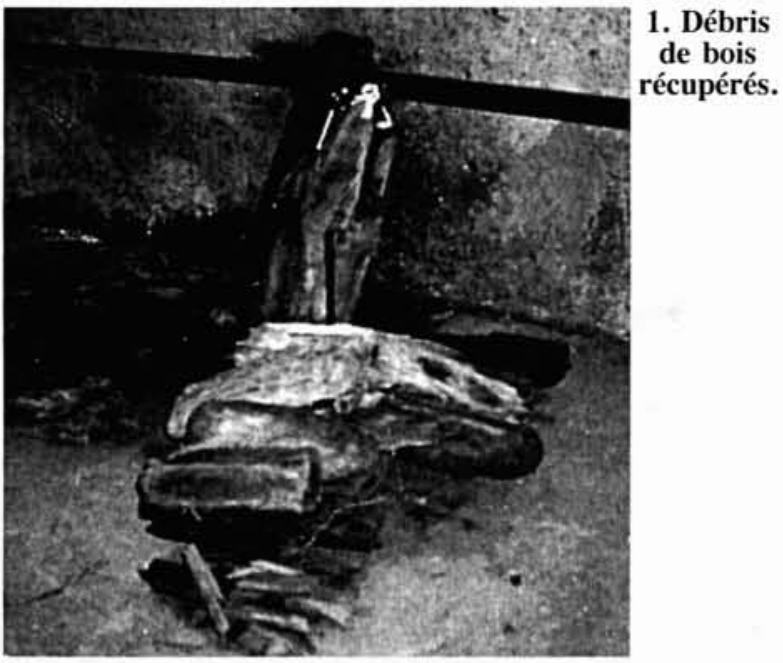

Un exemple, sur la station de ChongQing en Chine : l'eau brute du Yangtsé alimente des pompes centrifuges à aspiration axiale verticalisées. Le débit est conséquent, $1,33 \mathrm{~m}^{3} / \mathrm{s}$ par pompe, soit $4 \mathrm{~m}^{3 / \mathrm{s}}$ au total. Chaque moteur a une puissance de $1400 \mathrm{~kW}$. Il y a $200 \mathrm{~m}$ de conduite d'aspiration jusqu'au poste de pompage. Malgré le nettoyage initial, des morceaux de bois en quantité, dont le plus gros faisait $0,6 \mathrm{~m}$ x $0,15 \mathrm{~m} \times 0,15 \mathrm{~m}$ ont été retrouvés dans la pompe et le clapet. Il a donc fallu tout nettoyer une nouvelle fois et s'assurer que ce nettoyage a été fait correctement.

En Indonésie, sur l'affaire de Cisadane, des sachets plastiques ont été retrouvés en grande quantité dans les 6 pompes de $0,85 \mathrm{~m}^{3} / \mathrm{s}$ de débit unitaire. Il était impératif d'arrêter les pompes après 6 heures de marche continue pour les nettoyer.

Cela n'était pas perceptible au vu de la surface de la rivière, mais il $\mathrm{y}$ a une explication très claire, car les habitants de Tangerang et des localités voisines ont pour habitude d'acheter leur nourriture en ville servie dans des petits sachets plastiques, qu'ils jettent par terre dans les fossés, le tout se rejetant dans la rivière la première pluie venue. Le problème n'a bien sûr pas été mis en évidence au démarrage des études de l'affaire, ni d'ailleurs pendant le chantier.

Que faire? Deux passes de dégrillage fin droit (classique) avaient été construites en Génie Civil, mais les équipements, qui étaient en option, n'avaient pas été retenus par le client.

Une autre conception de la prise d'eau a été étudiée mais le résultat a été négatif car il a été bien établi que les sachets plastiques, ayant la même densité que l'eau, flottaient entre deux eaux et à aucun moment ne flottaient en surface ou ne décantaient au fond de la rivière.

La solution préconisée par Degrémont a consisté à faire installer le dégrillage prévu.

Il a fallu un an pour trouver la solution après qu'un tamisage manuel ait été mis en place ; le nettoyage du tamisage provisoire était fait par des plongeurs indonésiens.

\section{III — LES CARACTÉRISTIQUES DES POMPES}

Le type des pompes est dépendant des caractéristiques hydrauliques de l'installation, mais également de l'application des cahiers des charges contractuels sur les affaires.

Pour la définition du matériel, l'aspect compétitivité est essentiel car il importe de concevoir une installation avec le meilleur rapport qualité/prix. Toutefois il ne s'agit pas de fournir un matériel qui est conforme au cahier des charges sans s'être assuré du futur bon fonctionnement du matériel.

Il convient donc qu'avec les fournisseurs de pompes un bon climat s'établisse quant à la conception des postes de pompage.

Il y a plus de dix ans, on pouvait considérer tous les “ pompistes" comme des spécialistes de l'ingénierie du pompage. Leur technicité et leurs conseils permettaient de concevoir en commun des installations performantes pour lesquelles très peu d'anomalies étaient constatées.

Aujourd'hui il n'en va plus de même, l'aspect compétitivité a dépassé l'aspect technique et le matériel, qui a été optimisé, peut dans certains cas ne pas répondre aux attentes.

Nous allons en donner quelques exemples ci-après.

\section{- 3.1 L'aspiration}

Les aspirations de pompage sont souvent délicates à étudier dans le cas des pompages d'eaux brutes dans les fleuves et rivières. Il faut en effet faire transiter l'eau depuis un plan d'eau en général incontrôlé vers une bâche de pompage.

Un certain nombre de solutions techniques innovantes sont quelquefois demandées.

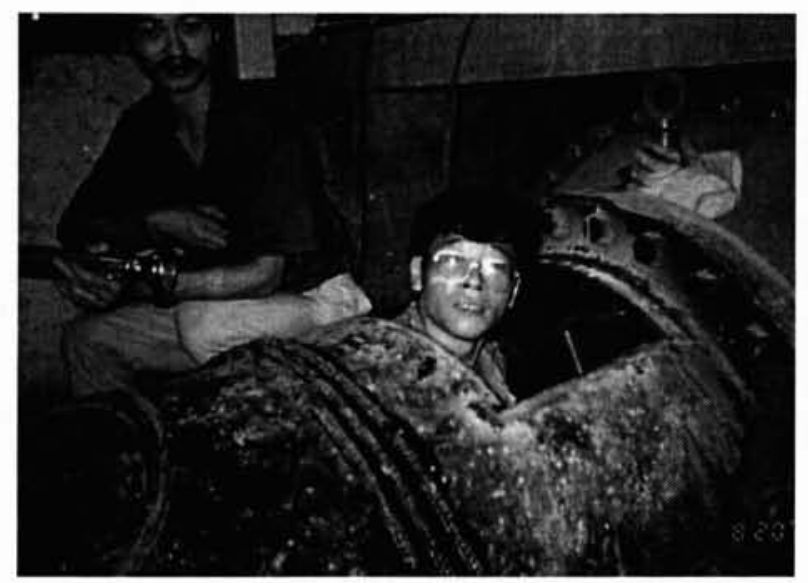

2. Percement de la conduite de refoulement

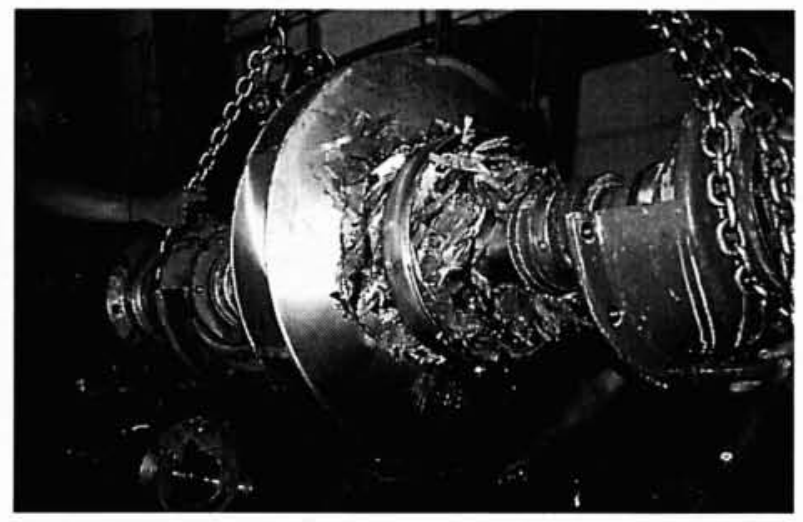

3. Extraction d'un arbre de pompe avec les sacs plastiques. 


\section{pplications industrielles de la mécanique des fluides}

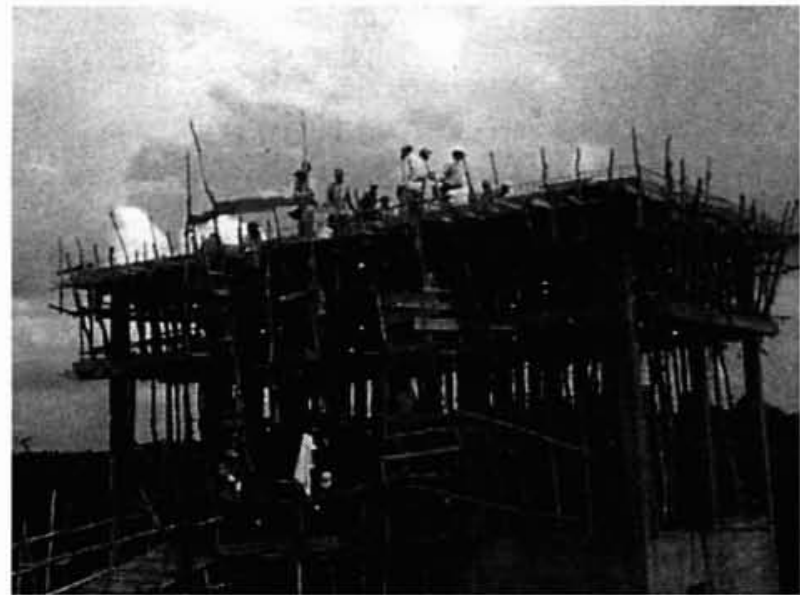

4. Têtes de pieux sortant de la rivière.

Il faut en faire le tri et préciser les critères hydrauliques pour une conception correcte.

Le niveau de la rivière est essentiel, c'est lui qui fixe les hauteurs géométriques minimale et maximale pour le pompage.

Ainsi sur la station de pompage de CAN THO au Vietnam, le pompage était prévu directement en rivière, dans le lit d'un affluent du Mékong. Après que les pieux aient été battus, une crue maximale de la rivière a emporté une partie de la rive concernée, ce qui a conduit à concevoir un nouveau poste de pompage dans les terres protégées des crues.

Les trois pompes verticales de débit $0,3 \mathrm{~m}^{3} / \mathrm{s}$ ont été conservées, mais du fait des nouvelles conditions d'installation, un élément de colonne a été supprimé, et il a fallu recalculer le NPSH disponible de l'installation et vérifier s'il était bien compatible avec celui de la pompe.

A Cavado au Portugal, les pompes étaient prévues " flottantes " dans le lit de rivière. La pompe prescrite était une pompe immergée type tube placée dans la conduite de refoulement.

La conception a évolué vers des pompes verticales lors

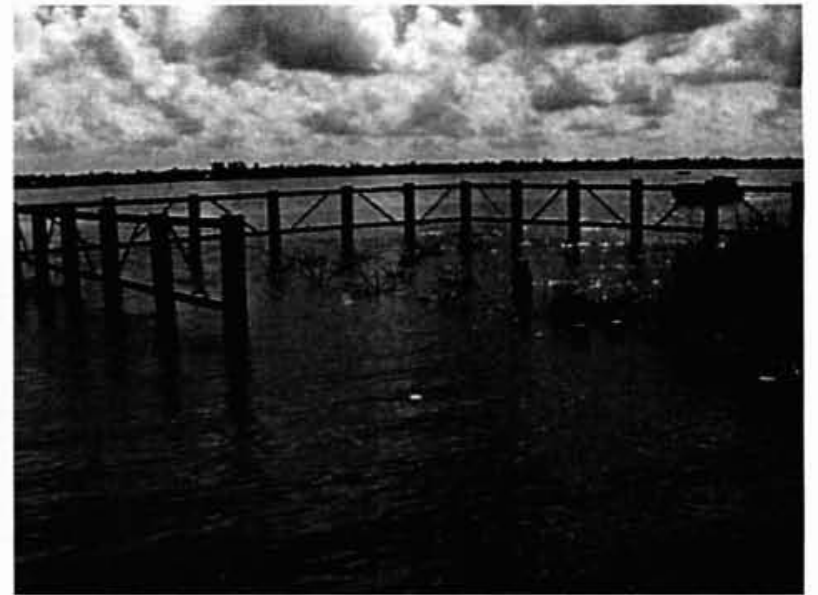

5. Le nouveau bâtiment de pompage.

des études de réalisation.

\section{- 3.2 Le NPSH}

Lorsque l'on parle de NPHS, on pense à la cavitation et au fait qu'il y a une limite basse du niveau bas dans les bâches d'aspiration en deçà de laquelle il est interdit de fonctionner, sinon il y a risque de destruction de la pompe.

Chaque cas doit être vérifié pour comparer si le NPSH disponible de l'installation est bien compatible avec le NPSH requis de la pompe. C'est le fournisseur de la pompe qui impose la différence entre ces deux valeurs et en prend la responsabilité.

Il faut toutefois remarquer que la valeur du NPSH requis (à $3 \%$ ) varie avec le type de roue proposé par le constructeur, mais également avec la nature du matériau de la roue. En effet on va trouver des différences importantes pour un même type de roues entre les roues en fonte, bronze et acier inoxydable, et il s'agit de valeurs supérieures à $1 \mathrm{~m}$.

La conséquence est que la pompe la plus compétitive en prix, c'est-à-dire avec une roue en fonte, doit être installée à un niveau plus bas, et cela coûte bien souvent très cher en

6. Installation projetée initialement sur la rivière Cavado.

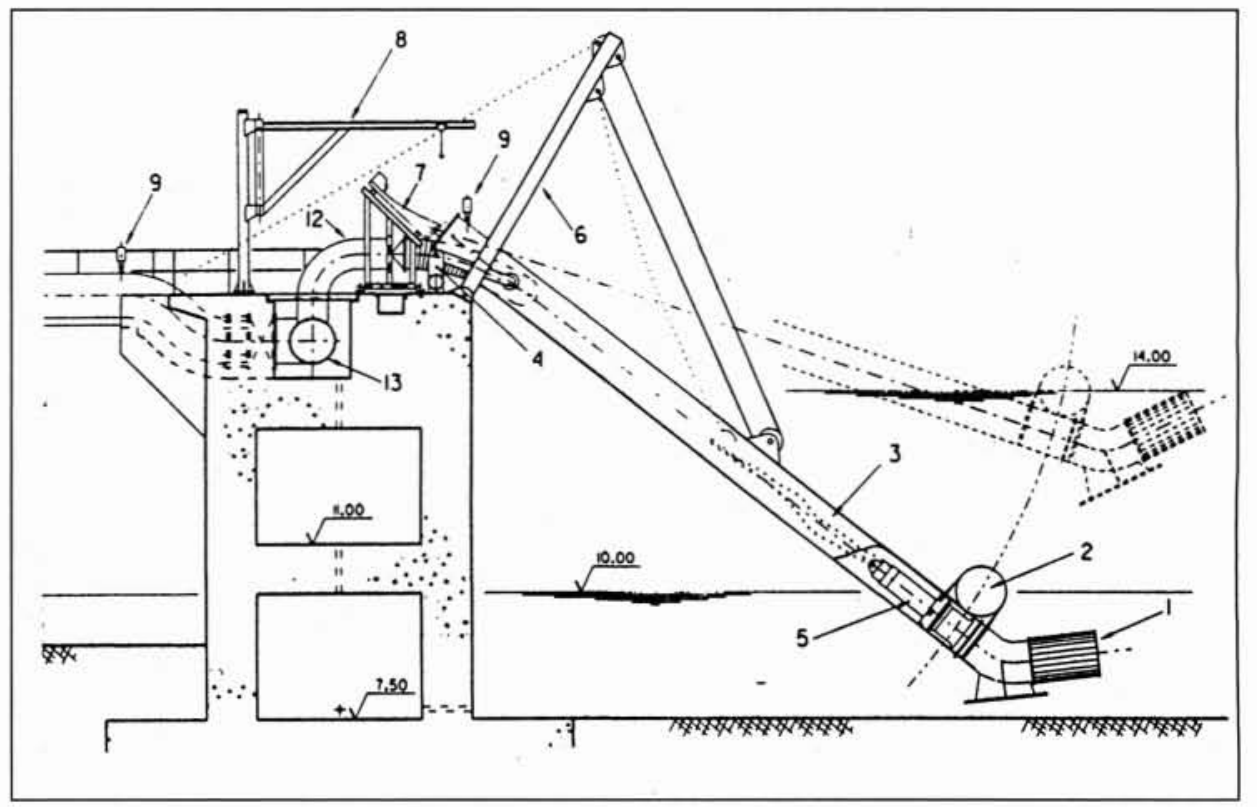


Génie Civil car il faut enterrer les ouvrages, et qui plus est, on se retrouve souvent dans la nappe phréatique.

Par ailleurs, il est très rare d'obtenir avec l'offre du fournisseur une courbe de NPSH qui couvre la totalité de la courbe hauteur/débit. Là encore un effort est à faire et il a été demandé à l'AFNOR d'intégrer cette demande dans la prochaine mise à jour de la norme correspondante NF EN ISO 9905 (E44-152).

\section{- 3.3 La pression de refoulement}

II n'est pas rare de constater dans les cahiers des charges des pressions de refoulement élevées, supérieures à 4 ou 5 bars.

Lors des études, il est important de s'assurer que le réseau existant est bien conçu et peut supporter la pression de la pompe. En effet le proverbe " Qui peut le plus peut le moins " ne s'adapte absolument pas dans le cas des pressions dans les réseaux.

Si pour les pompes centrifuges la pression de la pompe suit sa courbe et s'adapte à la pression du réseau, il faut vérifier que la pression minimale convient bien et n'est pas à l'extérieur de la plage de fonctionnement normale de la pompe.

Un exemple :

sur la station de Sam Sen, les équipements ont été installés avec la pression contractuelle prévue au cahier des charges, 4 bars. En réalité, le réseau supportait une pression entre 0,4 et 1,7 bars environ. La seule manière de baisser la pression a été de diminuer la vitesse de rotation de la pompe et de créer une perte de charge singulière en fermant partiellement les vannes au refoulement des pompes. Dans le cas contraire, le débit unitaire de la pompe augmente jusqu'à atteindre son débit maximum et dépasser la plage normale de fonctionnement.

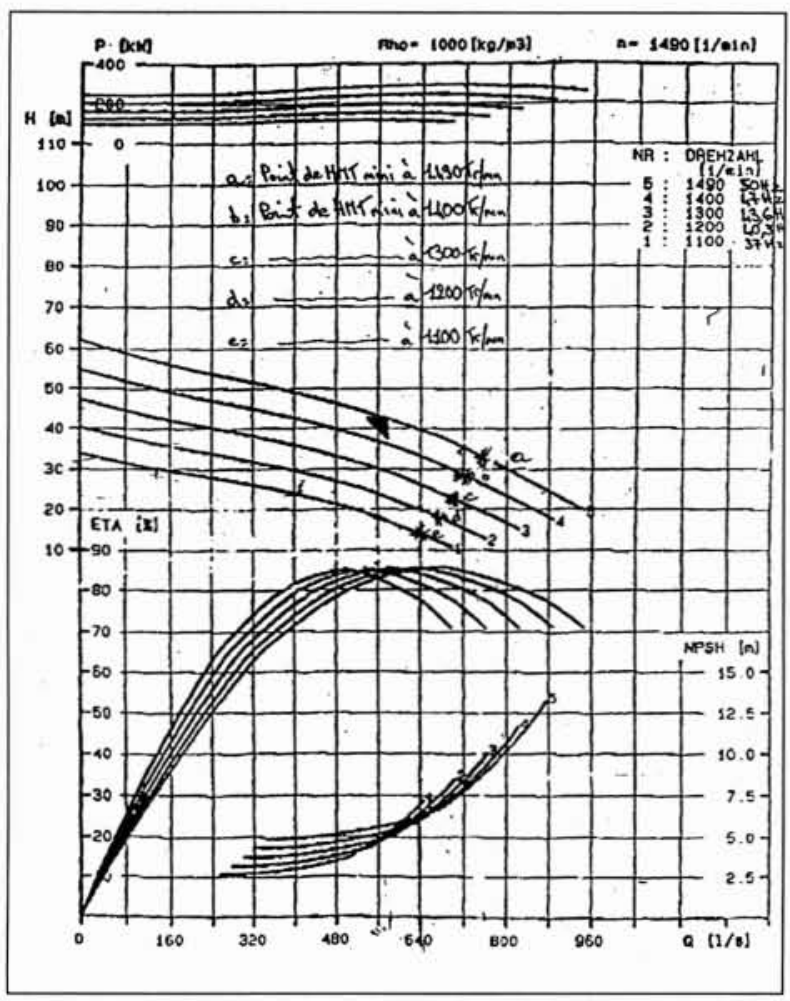

7. Courbes de fonctionnement des pompes.
On remarquera le point initial de fonctionnement à plus de $40 \mathrm{mCE}$.

On précise qu'à la vitesse minimum de $1100 \mathrm{tr} / \mathrm{min}$, on peut fonctionner avec une pression inférieure à $16 \mathrm{mCE}$.

On voit que le rendement des pompes baisse et surtout que le NPSH requis est fortement augmenté de plusieurs mètres.

\section{- 3.4 Les régimes transitoires}

Les coups de bélier et coups de clapet sont deux conséquences habituelles des régimes transitoires qui apparaissent lors des mises en service et des arrêts des installations soit automatiques, soit par coupure de courant électrique.

Il est souvent demandé la fourniture d'une protection antibélier. Les habitudes en l'absence de la connaissance du profil en long de réseau de distribution d'eau potable, sont d'abord de protéger les pompes. Les calculs sont faits avec la collaboration des fournisseurs d'anti-bélier ou de pompes en prenant des hypothèses qui sont à vérifier sur le site avec le client.

De manière à aller plus vite dans l'analyse, Degrémont s'est doté d'un logiciel anti-bélier et peut lors de ses études faire des simulations rapidement.

Dans certains cas il est toutefois nécessaire de faire appel à des sociétés spécialistes qui peuvent apporter des compétences complémentaires, particulièrement sur les réseaux d'eau potable.

\section{L L'INGÉNIERIE DU POMPAGE}

Depuis plusieurs décennies, Degrémont réalise des stations de pompage avec la collaboration des fournisseurs de pompes.

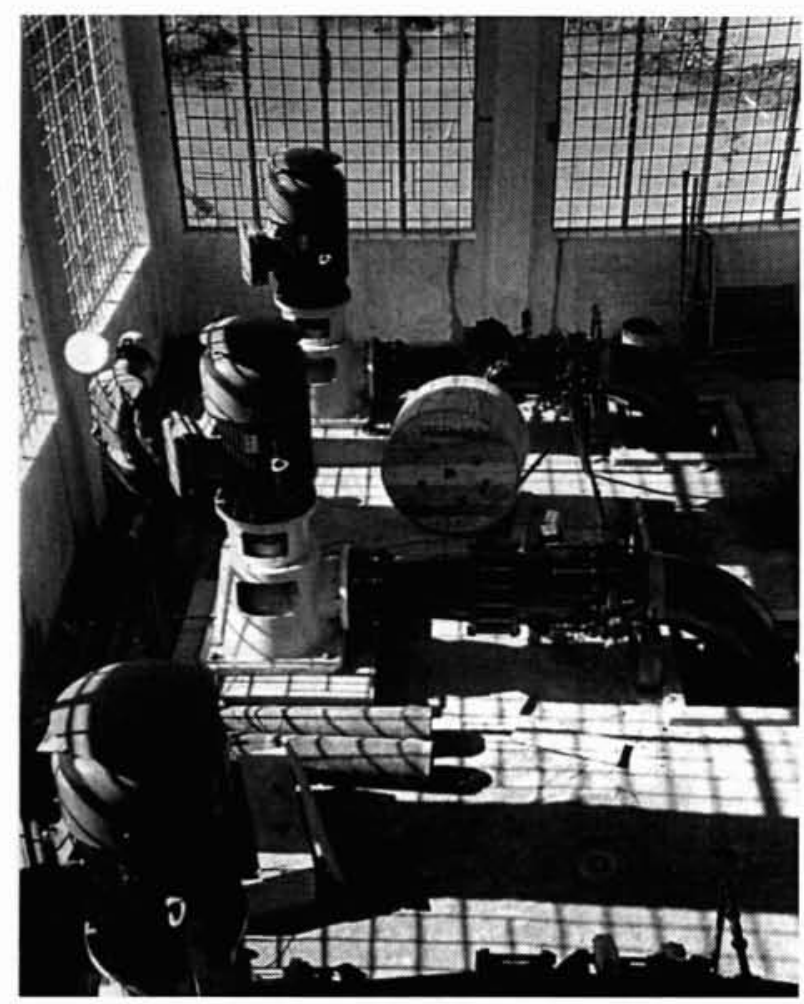

8. Station de pompage de Chongqing. 


\section{pplications industrielles de la mécanique des fluides}

Ainsi de grandes et belles installations ont été conçues et mises en service dans le monde sans problème particulier. Nous en citerons quelques-unes comme Maha Sawat en Thailande (pompes de $2,5 \mathrm{~m}^{3} / \mathrm{s}$ et moteurs de $1500 \mathrm{~kW}$ ), Adiyan au Nigéria (pompes de $3,7 \mathrm{~m}^{3} / \mathrm{s}$ et moteurs de $1400 \mathrm{~kW}$ ), Grapham et Hanningfield en Angleterre (pompes de $1 \mathrm{~m}^{3} / \mathrm{s}$ ), Mostorod en Egypte, Chongqing en Chine (pompes de $1,3 \mathrm{~m}^{3} / \mathrm{s}$ et moteurs de $1400 \mathrm{~kW}$ )...

L'ingénierie s'est faite naturellement entre les fournisseurs et les bureaux d'études de Degrémont.

Aujourd'hui pour certains fournisseurs l'aspect compétitivité a conduit d'une part à proposer un matériel optimisé en coût donc également en conception, et dans certains cas, nous avons atteint la limite à partir de laquelle des soucis sérieux sont apparus, et dont quelques exemples ont été développés ci-avant.

Cela a conduit Degrémont à se spécialiser et à investir dans l'ingénierie de pompage de manière interne, à s'obliger à contrôler les documents des fournisseurs, à mieux contrôler le matériel. Ainsi des logiciels pour la modélisation informatique, pour les calculs anti-bélier ont été acquis et une formation technique a été apportée aux ingénieurs de bureaux d'Etudes sur l'aspect pompage.

\section{$V \square$ CONCLUSION : L'AVENIR}

Cet effort interne ne doit pas cacher son origine et l'objectif est de pouvoir faire confiance aux fournisseurs de pompes, qui eux-mêmes souhaitons-le auront à cœur de pro- poser des équipements compétitifs, mais en s'étant assurés qu'il restait une marge technique suffisante pour que les problèmes rencontrés ne se reproduisent plus, tant dans les phases études que dans les phases réalisation.

\section{BIBLIOGRAPHIE}

[1] Les stations de pompage d'eau, AGHTM, éditions Lavoisier (TEC \& DOC)

[2] Hydraulique urbaine, tome II, A. Dupont, éditions Eyrolles

[3] Les installations de pompage - conception et réalisation publié par l'Association Française pour l'Etude des Eaux

[4] Guide technique pour l'installation des matériels de pompage, publić par la Chambre Syndicale Nationale des Entreprises et Industries de l'Hygiène Publique et le Syndicat des Constructeurs de Pompes.

[5] Les pompes et les petites stations de pompage, publié par SOGREAH

[6] Stations de pompage, publié J. Launay et G. Perrotin, extrait des Techniques de l'Ingénieurs

[7] Centrifugal pumps - Design operating principles, par Pompes Guinard, éditions Nathan International

[8] Norme E44-203/E44-152, AFNOR 